\title{
406. Die gastro-duodenale Ulcusblutung: Ergebnisse eines Therapiekonzeptes nach endoskopischen Kriterien
}

\author{
H. Stöltzing, K. Thon, W. Dietz, Ch. Ohmann, W. Lorenz und H. D. Roher
}

Zentrum für Operative Medizin I, Chirurgische Klinik und Abteilung für Theoretische Chirurgie der Philipps-Universität Marburg, Robert-Koch-Straße 8, D-3550 Marburg/Lahn

\section{Bleeding From Gastroduodenal Ulcer: Results with a Therapeutic Concept Based on Endoscopic Criteria}

\begin{abstract}
Summary. From 1 January 1982 to 31 March 1983, in a prospective study 64 patients with bleeding from gastroduodenal ulcers were treated according to a new therapeutic concept based mainly on the findings at emergency endoscopy with due consideration of bleeding activity and bleeding type. In the case of ulcers with arterial bleeding (stage 1A) surgery was performed after stabilization of the circulation. Since the introduction of this new therapy regimen the mortality in cases of bleeding gastroduodenal ulcer has fallen from $15 \%$ to $4.7 \%$. Of 23 patients subjected to surgery, 1 died (operative mortality $4.3 \%$ ).
\end{abstract}

Key words: Upper gastrointestinal bleeding - Emergency endoscopy.

Zusammenfassung. Vom 1. 1. 1982-31.3. 1983 wurden 64 Patienten mit einer gastro-duodenalen Ulcusblutung im Rahmen einer prospektiven Studie nach einem neuen Therapiekonzept behandelt, das sich überwiegend nach dem notfallendoskopischen Befund unter Berücksichtigung von Blutungsaktivität und Blutungstyp richtete. Hierbei wurden Ulcera mit arterieller Blutung (Stadium $1 \mathrm{~A}$ ) nach Kreislaufstabilisierung im Regelfall operiert. Die Gesamtletalität betrug beim Ulcus ventriculi $6,4 \%$, beim Ulcus duodeni $3 \%$. Von 23 operierten Patienten verstarb einer (OP-Letalität 4,3\%).

Schliisselwörter: Obere Gastrointestinalblutung - Notfallendoskopie - Gastro-duodenales Ulcus.

\section{Endoskopische prognostische Beurteilung der Blutungsneigung von Ulcera ventriculi und Ulcera duodeni}

\author{
K.-H. Fuchs und H.-J. Wirtz \\ Chirurgische Universitätsklinik Kiel, Abteilung Allgemeinchirurgie, Hospitalstraße 40, D-2300 Kiel

\section{Endoscopic Evaluation of Bleeding Peptic Ulcers for Prediction of Recurrent Hemorrhage}

\begin{abstract}
Summary. The clinical course of 121 patients with bleeding peptic ulcers was reviewed (1981/82). Recurrent hemorrhage, operation frequency and mortality rate were related to different bleeding types using a modified Forrest classification. Endoscopic criteria for predicting recurrent hemorrhage were evaluated. The results show that emergency endoscopy is a valuable method not only for accurate diagnosis, but also for prediction of recurrent bleeding from gastroduodenal ulcers.
\end{abstract}

Key words: Emergency endoscopy - Gastroduodenal ulcers - Recurrent bleeding - Possibility of forecasting.

Zusammenfassung. Der klinische Verlauf von 121 Patienten mit blutenden gastroduodenalen Ulcera wurde untersucht (1981/82). Blutungsrezidivhäufigkeit, Operationshäufigkeit und Letalität wurden mit den verschiedenen Blutungstypen einer modifizierten Forrest-Einteilung korreliert. Endoskopische Kriterien für die prognostische Beurteilung der Blutungsrezidivhäufigkeit wurden herausgearbeitet. Die Resultate zeigen, daß die Notfallendoskopie bei Ulcusblutung nicht nur die Diagnose, sondern darüber hinaus eine prognostische Beurteilung des Verlaufs ermöglicht.

Schliisselwörter: Notfallendoskopie - Gastroduodenale Ulcera - Blutungsrezidivhäufigkeit - Prognose. 